\title{
RursuSpicae
}

Transmission, réception et réécriture de textes, de

I'Antiquité au Moyen Âge

$3 \mid 2020$

La conversation des encyclopédistes

\section{Des textes sources au texte compilé : le portrait de l'autruche dans les compilations naturalistes des ordres mendiants au XIII ${ }^{\mathrm{e}}$ siècle}

From Sources to Compilations: Portraying the Ostrich in the 13th-Century

Compilations about Nature of the Mendicant Orders

\section{Grégory Clesse}

\section{OpenEdition}

Journals

Édition électronique

URL : http://journals.openedition.org/rursuspicae/1486

DOI : 10.4000/rursuspicae. 1486

ISSN : 2557-8839

Éditeur :

Université Nice-Sophia Antipolis, IRHT - Institut de recherche et d'histoire des textes

Référence électronique

Grégory Clesse, «Des textes sources au texte compilé : le portrait de l'autruche dans les compilations naturalistes des ordres mendiants au XIII e siècle », RursuSpicae [En ligne], 3 | 2020, mis en ligne le 30 décembre 2020, consulté le 31 janvier 2021. URL : http://journals.openedition.org/rursuspicae/1486 DOI : https://doi.org/10.4000/rursuspicae.1486

Ce document a été généré automatiquement le 31 janvier 2021.

RursuSpicae 


\section{Des textes sources au texte compilé : le portrait de l'autruche dans les compilations naturalistes des ordres mendiants au XIII ${ }^{\mathrm{e}}$ siècle $^{1}$}

From Sources to Compilations: Portraying the Ostrich in the 13th-Century

Compilations about Nature of the Mendicant Orders

Grégory Clesse

Elle pond ses œufs au mois de juillet et les cache dans le sable. Ceux-ci éclosent par la chaleur du soleil, comme de nombreux autres œufs d'animaux. Et c'est pourquoi elle n'y revient pas, car elle ne peut les couver de son corps nu; et (de temps en temps) elle surveille l'endroit où ils ont été cachés. De là, certains ont cru à tort qu'elle les couve du regard. [voir] Albert, Sur les animaux, livre $23^{2}$. 


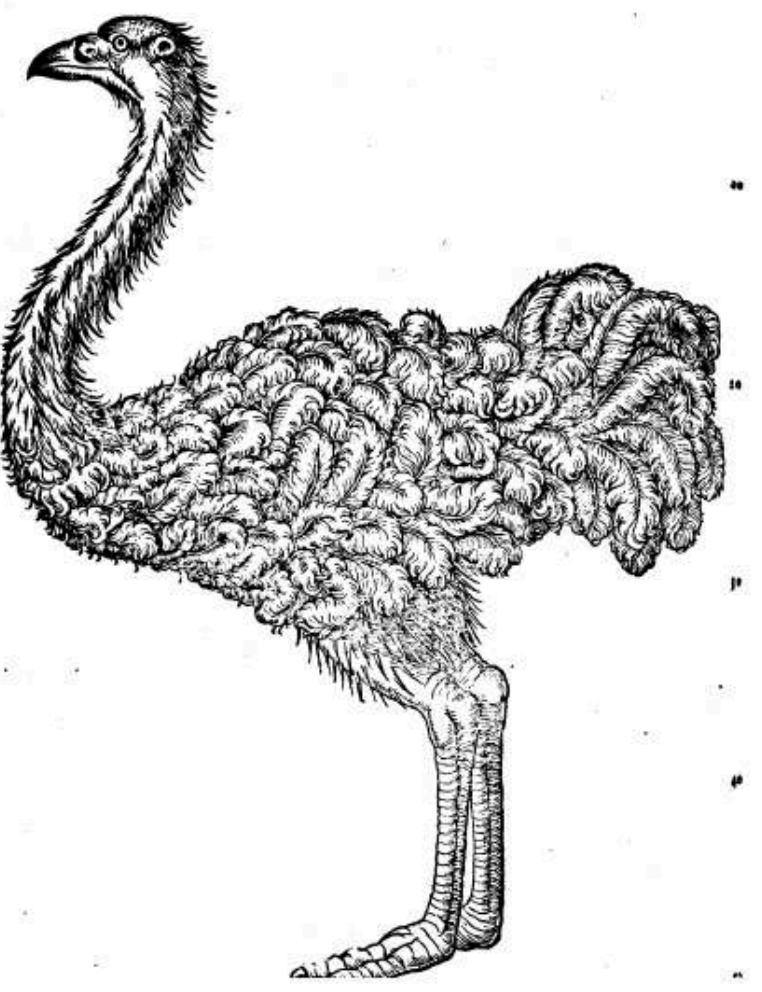

FIG. 1. Conrad Gesner, Historiae Animalium Liber III, qui est de Avium natura, éd. Ioannes Wechelus, Frankfurt, 1585, p. 740

1 Ce passage tiré de l'Historia animalium de Conrad Gesner reflète le développement progressif de la zoologie à partir de lignes de continuité clairement revendiquées avec la tradition ancienne et médiévale. Afin d'appréhender cette chaîne de transmission, nos récents travaux ont abordé l'examen des sources encyclopédiques en partant du savoir disciplinaire pour aborder ensuite l'étude des textes compilés ${ }^{3}$. Cette perspective prend le contre-pied des analyses habituelles, qui s'appuient plutôt sur les citations d'autorités pour ensuite «remonter le courant» vers les sources utilisées par le compilateur ${ }^{4}$. Dans cet article, nous prolongeons l'essai de façon plus aboutie avec un cas d'étude spécifique. Dans un premier temps, nous passons en revue les informations accessibles au milieu du XIIIe siècle sur un sujet donné. Dans un second temps, nous comparons ces contenus avec les synthèses sur la nature réalisées dans les ordres mendiants au cours du XIIIe siècle. Pour ce faire, il convient de cibler un thème qui soit traité par un éventail d'auteurs suffisamment représentatif, tout en veillant à ce que ce tour d'horizon reste dans des proportions maîtrisables. Selon ces critères, nous avons choisi d'analyser les savoirs sur l'autruche (strutio ou assida) dans les textes sources et dans les synthèses du XIIIe siècle, en focalisant l'attention sur le Liber de natura rerum de Thomas de Cantimpré, le De proprietatibus rerum de Barthélemy l'Anglais, le Speculum naturale de Vincent de Beauvais et le Liber de animalibus d'Albert le Grand. Plusieurs enjeux sont inhérents à cette perspective de recherche, en particulier le statut des compilations sur la nature par rapport au reste de la tradition, la question de la disponibilité des sources dans les bibliothèques accessibles à chacun des compilateurs et de la sélection d'informations issues de divers champs disciplinaires dans ces sources. L'information médiévale sur l'autruche peut ainsi être analysée non seulement à partir des contenus retenus par les compilateurs, mais aussi en fonction de ceux qu'ils ont préféré écarter. 
2 Dans le domaine zoologique, le compilateur latin du XIIIe siècle disposait en effet d'une vaste documentation. Parmi les sources à sa disposition, une attention spécifique doit d'abord être réservée aux auteurs suivants, dont l'œuvre se caractérise par un effort de synthèse encyclopédique ${ }^{5}$ : Aristote (384-322 av. J.-C.), Pline (23-79 ap. J.-C.), Isidore (v. 560-570 - 636), Raban Maur (v. 780-856), Lambert de Saint-Omer (975-1043), Alexander Neckam (1157-1217) et Gervais de Tilbury (v. 1152 - v. 1234) ${ }^{6}$. La littérature moralisée des bestiaires constitue un deuxième canal de diffusion des savoirs zoologiques ${ }^{7}$. Dans ce registre, nous avons examiné les ouvrages-sources suivants: le Physiologus B (IIe ou IVe s.), les Dicta Chrysostomi (v. 1000) et l'Aviarium d'Hugues de Fouilloy (m. 1173/4) . Cet aperçu est également complété par un sondage au cœur des Moralia in Iob de Grégoire le Grand (v. 540-604), de la Glossa ordinaria (XIIe s.) et de quelques recueils de propriétés qui servaient d'outils de rédaction aux prédicateurs ${ }^{9}$ : Angelus attribué à Garnier de Rochefort (v. 1140-après 1226), Angelus purus (première moitié du XIIIe s.), Abies arbor alta (première moitié du XIIIe s.), Anonyme de Clairvaux (deuxième tiers du XIIIe s.) ${ }^{10}$. Il convient enfin de prendre en compte le domaine médical et pharmacologique, en explorant les traductions latines d'auteurs arabes comme le Liber ad Almansorem de Rāzī (865-925), le De dietis d'Isaac Israeli (v. 830-850v. 932-955), le Canon et l'Abbreviatio de animalibus d'Avicenne (980-1037) ${ }^{11}$, ou encore les écrits d'Hildegarde de Bingen (1098-1179). Le Pantegni de 'Alī ibn al-'Abbās al-Majūsī (d'après Galien), dans sa version latine par Constantin l'Africain (v. 1020-1087) n'a, quant à lui, pas apporté d'éléments significatifs relatifs à l'autruche. En annexe, un tableau comparatif reprend l'ensemble des contenus sur l'autruche rapportés par ces différentes sources.

\section{Bestiaires, encyclopédies, recueils de propriétés et traités scientifiques : typologie des informations sur l'autruche dans les textes sources}

3 Parmi les informations recueillies dans ce vaste corpus, nous trouvons tout d'abord des considérations sur la dénomination latine de l'animal et son étymologie. Le Physiologus B relève une double appellation, asida et strutiocameleon en grec, faisant écho à la similarité des pattes de l'autruche avec les pieds du chameau ${ }^{12}$. Cette présentation est reprise dans les Dicta Chrysostomi ${ }^{13}$. Isidore de Séville mentionne quant à lui une étymologie grecque faisant allusion au fait que l'autruche semble avoir des plumes à l'instar d'un oiseau, bien qu'elle ne puisse s'élever de terre ${ }^{14}$. Cette étymologie est reprise ensuite par Raban Maur ${ }^{15}$. Notons déjà que, dans le Canon d'Avicenne, l'autruche est rangée sous la notice de naham (Liber Canonis, II, 526), translittération de l'arabe (na'ām), qui désigne plus spécifiquement la femelle, tandis que le mâle fait l'objet d'un chapitre de thalio (II, 721, translittéré de l'arabe - ظَalìm) qui renvoie seulement à la notice de structione, c'est-à-dire, en réalité, au chapitre de naham ${ }^{16}$.

4 Une autre question discutée dans les textes sources concerne la classification de l'espèce, assimilée aux oiseaux mais présentant des similitudes avec certains quadrupèdes. Aristote et Avicenne (dans l'Abbreviatio de animalibus) se penchent particulièrement sur ce point. Ainsi, Aristote (PA, III, 14, 697b 14-26) ${ }^{17}$ évoque la présence d'ailes qui ne permettent toutefois pas à l'autruche de voler et qui sont plutôt semblables à des poils. La grosseur de l'autruche la rapproche d'un quadrupède, tout comme ses cils au-dessus des paupières supérieures, sa tête pelée et le sommet de son 
cou. Ses deux pattes, couvertes de plumes à l'instar des oiseaux mais se terminant par deux pinces (et non des doigts) comme chez certains quadrupèdes, sont une autre caractéristique de cette ambiguïté oiseau-quadrupède. Avicenne s'inscrit dans une même lignée : il met lui aussi l'accent sur la présence d'ailes, le nombre de pattes et la forme courbée du pied pour rapprocher l'autruche des oiseaux, et sur son duvet semblable à des poils et ses pattes terminées par une sorte de sabot fendu pour la rapprocher de quadrupèdes comme le chameau ${ }^{18}$.

D'autres auteurs ont développé la description physique de l'autruche sur la base de comparaisons avec d'autres animaux. Ils ont, eux aussi, porté leur attention sur sa grande taille: celle-ci dépasse celle d'un homme à cheval (Pline $\left.{ }^{19}\right)$, et cette disproportion du corps l'empêche de voler (Hugues de Fouilloy ${ }^{20}$ ). D'autres traits physiques ont retenu l'attention des auteurs: ses pattes, similaires à celles du cerf (Pline) ou à celles du chameau (Physiologus $\mathrm{B}^{21}$ ) ainsi que ses ailes, servant à la course plutôt qu'au vol (Pline), composées de rares plumes (Grégoire le Grand, Glose Ordinaire, Lambert de Saint-Omer, Hugues de Fouilloy, Angelus purus), semblables par leur couleur à celles du héron et de l'autour ${ }^{22}$ (Grégoire le Grand, Glose Ordinaire, Hugues de Fouilloy, Angelus, Angelus purus, Abies arbor alta, Anonyme de Clairvaux) ${ }^{23}$. D'un point de vue plus général, sa ressemblance avec le vautour est soulignée (Physiologus $\mathrm{B}^{24}$ ), tout comme sa nature chaude qui la rapproche des animaux sauvages (Hildegarde de Bingen ${ }^{25}$ ) et son excellente vision qui lui permet de retrouver ses petits cachés sous le sable (Alexander Neckam ${ }^{26}$ ).

6 Nous trouvons encore des renseignements sur le milieu géographique de l'autruche, situé en Afrique (Pline, Lambert de Saint-Omer) ou, plus précisément, en Éthiopie (Pline) voire en Libye d'après l'appellation «moineau de Libye» (Aristote) ${ }^{27}$. Par ailleurs, plusieurs informations ont trait au mode de vie et au comportement de l'autruche. Sa vitesse à la course, qui dépasserait celle d'un homme à cheval (Pline), est ainsi mise en avant (Pline, Hildegarde de Bingen). Ses pattes lui serviraient aussi à combattre ses poursuivants et à lancer des pierres (Pline ${ }^{28}$. Le perspicacité de l'autruche est également mise en exergue, à travers une anecdote rapportée par Gervais de Tilbury ${ }^{29}$, qui la reprend lui-même de Pierre le Mangeur ${ }^{30}$. Un jour, le roi Salomon aurait enfermé dans un vase le petit de son autruche. Celle-ci serait alors partie dans le désert en quête d'un vermisseau ayant la faculté de briser le verre, et c'est ainsi que Salomon eut lui-même connaissance de cette remarquable propriété du vermisseau (Gervais de Tilbury). Concernant son régime alimentaire, l'autruche serait capable de tout digérer (Pline), même le fer (Lambert de Saint-Omer, Alexander Neckam) $)^{31}$. Hildegarde de Bingen, quant à elle, assure qu'elle se nourrit plutôt d'herbe ${ }^{32}$. À propos de la reproduction, il est noté que l'autruche fait partie des oiseaux qui pondent le plus d'œufs (Aristote ${ }^{33}$ ). Cependant, il est très régulièrement souligné qu'elle ne couve pas ses œufs, mais les enfouit dans le sable (Isidore, repris ensuite par Raban Maur et Lambert de Saint-Omer; Physiologus B, repris dans les Dicta Chrysostomi; Grégoire le Grand ; Glose Ordinaire ; Hugues de Fouilloy ; Angelus; Angelus purus; Abies arbor alta; Hildegarde de Bingen $)^{34}$. Cette information commune prend néanmoins des colorations variées au sein de ces textes. Chez Isidore, elle conserve un caractère neutre et succinct. Chez Hildegarde, elle s'additionne d'une explication physiologique, à savoir que l'autruche est de nature trop chaude pour pouvoir couver ses œufs et qu'elle place donc ses œufs dans le sable, chaud et humide, avant que ses petits ne naissent et ne courent à ses côtés. Enfin, cette donnée factuelle est exploitée dans le Physiologus B et dans l'Aviarium, où elle sert d'appui à des interprétations moralisantes tantôt positives, 
tantôt négatives. Ainsi, le Physiologus B relate que l'autruche attend, avant de pondre, le lever de l'étoile Virgilia aux environs du mois de juin et oublie ensuite ses petits sous le sable. Ce développement de l'information offre plusieurs liens possibles avec des citations bibliques - Jérémie 8, 7 ; Job 39, 13-16 ; Matt. 8, 22 et 10, 37 ; Phil. 3, 14-14 -, mettant en exergue la capacité à se détourner du monde terrestre pour s'élever vers les choses célestes. À l'inverse, l'Aviarium investit l'autruche d'une ignorance de toute sollicitude maternelle, dans une présentation très négative de l'oiseau.

Abordons précisément les moralisations dont l'autruche fait l'objet, dans des directions assez diversifiées. Florence McCulloch avait déjà mis en avant l'interprétation fondée sur Jérémie 8, 7 et Job 39, 13-16 et selon laquelle l'autruche, qui dépose ses œufs et les fait éclore par la force de soleil, nous apprend à nous détourner du monde terrestre et à porter notre regard vers le monde céleste. Par contre, dans l'Aviarium et les recueils de propriétés, inspirés des Moralia in Iob et de la Glose ordinaire ${ }^{35}$, l'accent est placé sur l'incapacité de l'oiseau à voler, caractéristique qui nourrit une interprétation morale très négative ${ }^{36}$. Une telle ambivalence n'est pas rare : Baudouin Van den Abeele aboutit à un constat similaire pour d'autres oiseaux comme le merle, le paon et le faucon ${ }^{37}$, et souligne ainsi qu'« il y a en fait autant de sens possibles que l'animal a de propriétés évoquées par les auteurs $\aleph^{38}$. Une telle richesse allégorique se retrouve en partie dans le cas de l'autruche, mais le portrait qui en est brossé reste majoritairement négatif, à l'exception de ce que l'on trouve dans le Physiologus $\mathrm{B}^{39}$. Pline l'Ancien, déjà, associe l'autruche à la sottise, dans la mesure où elle pense être à couvert lorsqu'elle enfouit son cou dans la broussaille ${ }^{40}$. Par la suite, l'autruche est surtout assimilée à l'hypocrisie. L'argument principal repose sur le fait qu'elle possède des plumes, mais celles-ci ne lui assurent pas la capacité de voler (Grégoire le Grand, Glose Ordinaire, Hugues de Fouilloy, Anonyme de Clairvaux ${ }^{41}$. Une analogie peut aussi être établie avec les philosophes et les hérétiques, qui veulent s'exalter avec les plumes de la sagesse, mais restent incapables de voler (Raban Maur) ${ }^{42}$. Enfin, Alexander Neckam reprend l'image de l'hypocrite qui affiche un visage contemplatif, mais n'atteint pas le vol spirituel. Il ajoute encore que l'autruche consomme même le fer, qu'elle est dotée d'une excellente vision - qualité mise en relation avec l'hypocrisie - et qu'elle cache ce qui est clandestin pour exposer avec ostentation ce qui est public, en quête d'éloges venant d'autrui ${ }^{43}$.

Terminons ce panorama avec les indications sur les usages dérivés et les propriétés pharmacologiques relatifs à l'autruche. Ses œufs et ses plumes sont employés respectivement comme récipients et pour l'ornementation des casques (Pline) ${ }^{44}$. Sur le plan diététique, Hildegarde Bingen rapporte que la consommation de sa chair serait bénéfique contre le mal caduc (l'épilepsie) et aux hommes gras (et qu'elle est à l'inverse déconseillée pour les personnes maigres ou malades), car elle permettrait de fortifier le corps et d'éliminer les chairs superflues. Contre l'hydropisie (épanchement liquidien et œdème), Hildegarde fait aussi état d'une recette à base de coquilles d'œuf d'autruche mêlées à de l'eau, mais souligne par contre que les organes internes de l'autruche (cœur et poumon notamment) ne recèlent pas de vertu curative ${ }^{45}$. Parmi les auteurs arabes, Rāzī affirme que la chair d'autruche est estimée comme étant la plus grasse de toutes ${ }^{46}$. Isaac Israeli, quant à lui, caractérise la viande d'autruche par une humidité dominante. Dure et grasse, elle fatigue l'estomac et est la plus difficile à digérer. Par conséquent, on ne consomme que le cou et les ailes, bien que d'aucuns - Isaac s'en réfère ici à Galien louent la qualité de ses intestins. L'autruche est encore comparée avec le canard, dont la viande est jugée plus subtile et plus savoureuse ${ }^{47}$. Avicenne, enfin, rapporte dans son Canon que certains médecins louent la qualité de sa viande. Celle-ci, chaude et grasse, 
serait appétitive, favoriserait le coït et conforterait le corps lorsqu'elle est bien digérée, mais l'affaiblirait et ferait grossir dans le cas contraire ${ }^{48}$.

\section{L'autruche dans les notices du Liber de natura rerum de Thomas de Cantimpré}

Chez Thomas de Cantimpré ${ }^{49}$, le chapitre sur l'autruche débute par une citation attribuée à Isidore, mais dont il faut plutôt trouver l'origine dans le Physiologus. C'est d'ailleurs de cette source que provient toute la première partie de la notice (l. 1-13), parlant de l'étymologie ("camelon » car ses pieds sont semblables à ceux du chameau) et de la ponte. Le dominicain brabançon utilise sa source façon exhaustive. En effet, il évoque successivement les éléments suivants : 1) l'autruche attend le lever de l'étoile Virgilia avant de pondre ; 2) elle ensable ses œufs ; 3) elle est oublieuse par nature, se détache du terrestre pour obtenir la connaissance à travers la contemplation du ciel.

La deuxième partie (1. 14-33), ajoutée dans un second temps de rédaction, est davantage de l'ordre du raisonnement philosophique. En effet, une question initiale est posée : pourquoi l'autruche enfouit-elle ses œufs dans le sable et ne les récupère-t-elle qu'après leur éclosion? Ensuite, une solutio est envisagée. Comme l'a signalé Mattia Cipriani, cette addition provient des Questions salernitaines (Quest. Sal., N 62) ${ }^{50}$. Son contenu fait appel à différentes notions philosophiques, comme les causes matérielle et efficiente, la matière ou encore la substance. La réponse envisagée intègre des éléments présents chez certains des auteurs que nous avons passés en revue. L'explication selon laquelle l'autruche a une matière extrêmement grasse et produit donc une chaleur très élevée qui corromprait le processus de génération n'est pas sans rappeler Hildegarde de Bingen, qui décrivait aussi la nature chaude de l'autruche. On retrouve en outre certaines données plus factuelles bien attestées ailleurs dans la tradition, comme le fait que l'autruche soit capable de digérer le fer. Cette curiosité, rapportée par Lambert de Saint-Omer et Alexander Neckam à la suite de Pline, qui indiquait déjà que l'autruche mangeait de tout indistinctement, constituait une croyance partagée au XIIIe siècle, susceptible d'illustrer de façon concrète des débats intellectuels théoriques ${ }^{51}$.

11 La notice de Thomas de Cantimpré se poursuit par une phrase intégrée sans marqueur de citation, qui s'inscrit dans la continuité des Moralia in Iob, de la Glose Ordinaire, de l'Aviarium et des recueils de propriétés: « Hec avis pennas habet herodii et accipitris, sed est tarda ad volandum » (1. 34, «Cet oiseau a les plumes d'un héron et d'un autour, mais est lourde pour voler »). La suite de la citation, attribuée à Aristote (1.35-37) et qui porte sur la petite taille des plumes qui ne permettent pas à l'autruche de voler, semble plutôt typique des sources précédemment citées qui faisaient reposer la moralisation de l'autruche sur cet argument. Ensuite, la citation de l'Experimentator (1. 37-47) apporte plusieurs informations plus ou moins originales, la difficulté étant de déterminer la longueur de cette citation d'un ouvrage dont l'identification reste incertaine. Quoi qu'il en soit, dans la suite du texte, certains renseignements sont bien connus, comme l'ingestion de fer par l'autruche rendue possible par la nature très chaude de l'animal, ou encore la présence de paupières, mentionnée précédemment par Aristote. Par contre, dans les textes que nous avions parcourus, nous n'avions pas trouvé mention de son aversion pour le cheval ni du signalement d'un petit os qui serait situé sous ses ailes et mis en mouvement lors de crises de colère, caractéristiques qui sont quant à elles bien présentes dans l'Experimentator ${ }^{52}$. 
12 La fin de la notice (1. 48-52), inspirée de Pline, fait mention de la vitesse supérieure à celle du cheval, au jet de pierres contre les assaillants de l'autruche, à la stupidité de l'animal qui se croit invisible au moment d'enfouir son cou dans le sable, et à la confection de vases à partir de ses œufs. Par contre, nous n'avons pu retrouver l'origine du passage qui suit directement le marqueur de citation (1. 49-52) et qui informe de la perte naturelle de plumes laissant le corps de l'autruche quasiment à nu, de la résistance de l'enveloppe dermique qui protège l'animal du froid, de la présence d'un os allongé et large dans la poitrine qui protège l'autruche à la manière d'un bouclier, ou encore de l'indication de la taille de l'autruche comparable à celle d'un âne de dimension moyenne.

13 Cette analyse reflète l'intérêt marqué de Thomas de Cantimpré pour les curiosités qui ont trait à l'autruche, laissant de côté d'autres renseignements comme le milieu géographique de l'animal, son appartenance discutée au genre des oiseaux, ses caractéristiques physiques, son régime alimentaire, son mode de reproduction et ses propriétés pharmacologiques. L'exactitude des marqueurs de citations ne semble pas toujours avérée. Ainsi, alors qu'ils ne sont pas mentionnés, le Physiologus et la littérature morale ou spirituelle (Moralia in Iob, Glose Ordinaire, Hugues de Fouilloy et recueils de propriétés ultérieurs) ont sans conteste influencé le compilateur qui, comme on le sait, accorde une attention particulière aux moralisations $\mathrm{s}^{53}$. Enfin, certaines sources n'ont pas été privilégiées, comme Isaac Israeli, ou ne lui ont vraisemblablement pas été accessibles. C'est le cas du traité d'Hildegarde de Bingen, encore peu diffusé, et des traductions latines des livres de Rāzī et d'Avicenne, qui deviendront quelques décennies plus tard des ouvrages de référence dans le Speculum maius de Vincent de Beauvais et dans les universités de médecine ${ }^{54}$. Par conséquent, les informations de type diététique et pharmacologique ne se retrouvent pas dans le Liber de natura rerum, pas plus que l'anecdote relative à l'autruche du roi Salomon rapportée successivement par Pierre le Mangeur et Gervais de Tilbury. 


\section{L'autruche dans le De proprietatibus rerum de Barthélemy l'Anglais}

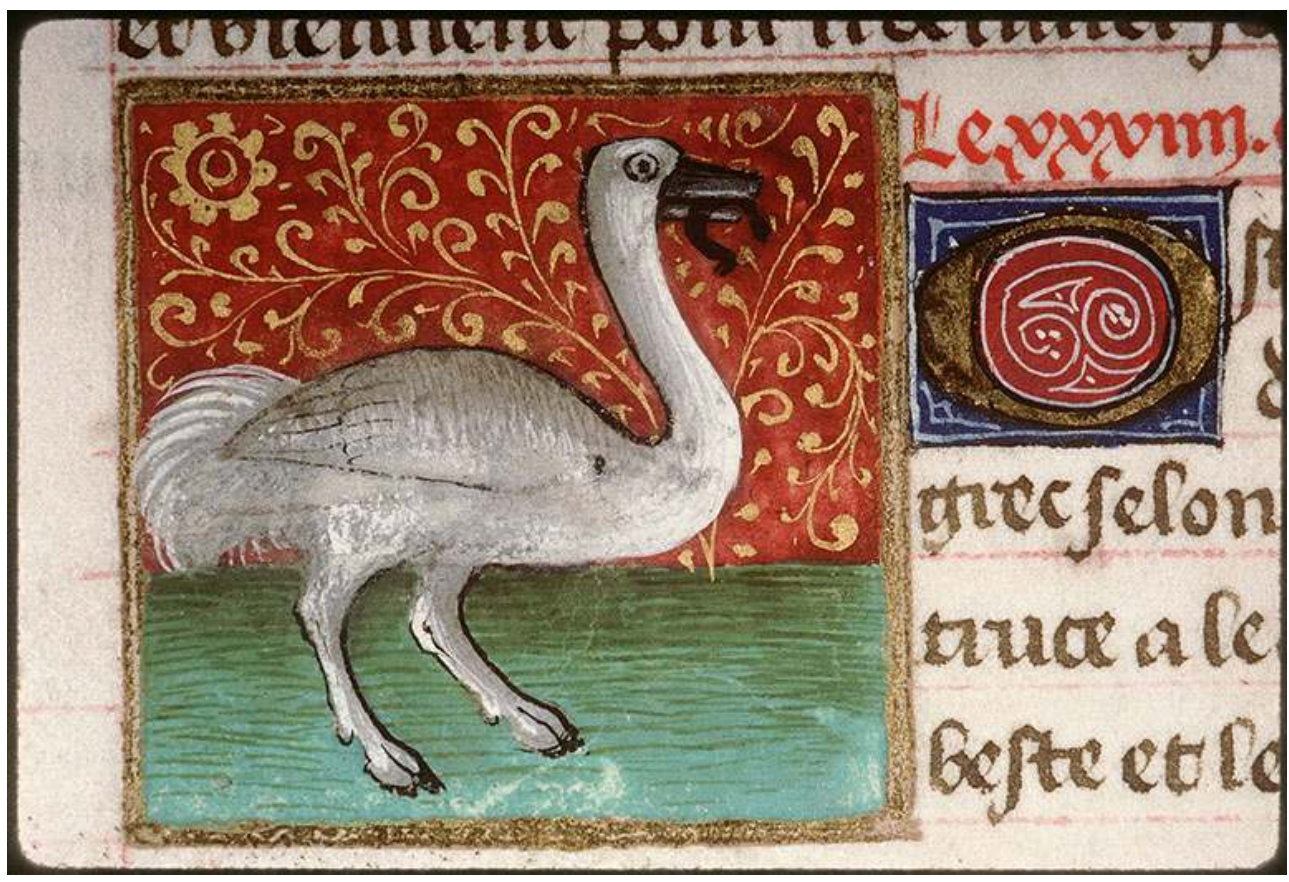

Fig. 2. Barthélemy l'Anglais, Livre des propriétés des choses (traduction française par Jean Corbechon), Amiens, BM, ms. 399, f. 147 v

Le Franciscain Barthélemy l'Anglais ${ }^{55}$ commence son chapitre sur l'autruche par la citation d'Isidore concernant l'étymologie du mot struthio, dans un passage qui rapporte déjà l'impossibilité de voler et l'ensablement des œufs (1.1-8). L'intégralité de l'extrait d'Isidore est repris, mais une addition est à souligner: " habet etiam duos pedes et rostrum sicut volucris » (" elle a deux pieds et un bec comme les oiseaux »). La mention des deux pieds rappelle Aristote et Avicenne, mais nous n'avons pas trouvé d'équivalent dans les textes sources pour celle du bec. Derrière une citation d'Isidore qui semble littérale à première vue, Barthélemy l'Anglais introduit en réalité un remaniement du texte qui lui est propre. Ensuite, Barthélemy l'Anglais reprend Aristote pour parler du classement de l'autruche dans la catégorie des oiseaux malgré les caractéristiques qui la différencient (1. 8-15). La suite de la notice est moins transparente. Sans spécifier de marqueur de citation, Barthélemy rapporte lui aussi le fait que l'autruche est capable de digérer le fer en raison de sa nature extrêmement chaude. Puis, il renvoie à Avicenne à propos de la grande taille et de la dureté des œufs, qui protègent le fotus d'une chaleur excessive au moment de son développement (l. 16-20). Malheureusement, nous n'avons pu retrouver le passage correspondant dans les textes sources.

La suite de la notice porte sur la ressemblance des plumes de l'autruche avec celles du héron, bien qu'elles ne lui permettent pas de voler en raison de son trop grand poids. Cette citation est attribuée à Grégoire le Grand (1. 20-27), mais a aussi pu être insérée à partir d'une source intermédiaire comme la Glose ordinaire, où le passage se retrouve également, voire l'Aviarium d'Hugues de Fouilloy, l'un des recueils de propriétés qui en sont aussi inspirés ou encore l'Experimentator ${ }^{56}$. Comme nous l'avons vu, ce passage a aussi été retenu par Thomas de Cantimpré. Puis, le texte évoque la génération au 
moment du lever de l'étoile Virgilia (1. 27-39) ; si la citation n'est pas littérale, elle fait sensiblement écho au texte du Physiologus, également repris par l'Experimentator et par Thomas de Cantimpré. Enfin, les dernières informations fournies par Barthélemy l'Anglais concernent l'aversion de l'autruche pour les chevaux, contre lesquels elle érige ses ailes pour les faire fuir (1. 39-43), renseignement que l'on trouvait aussi déjà dans l'Experimentator et chez Thomas de Cantimpré57.

La comparaison des deux compilateurs met en évidence la reprise d'informations communes et, à l'inverse, la mise à l'écart de certaines sources, en l'occurrence Pierre le Mangeur, Gervais de Tilbury, Hildegarde de Bingen, Rāzī, Isaac Israeli et le Canon d'Avicenne. Si la citation est correctement attribuée à Avicenne par Barthélemy l'Anglais, elle proviendrait plus probablement de l'Abbreviatio de animalibus, qui a été effectivement utilisée par le compilateur franciscain dans d'autres chapitres. Pline, très présent chez Thomas, n'est par contre pas utilisé par Barthélemy l'Anglais, qui structure plus explicitement la notice. Il fait le choix de débuter son propos par l'étymologie en y associant l'auteur source le plus pertinent et le plus spécifique dans ce registre : Isidore de Séville. Puis, il passe à des considérations sur la classification de l'autruche, utilisant là aussi l'auteur le plus approprié en la matière, à savoir Aristote, sans retenir les informations données par Avicenne sur ce même sujet. Enfin, les contenus suivants ressortissent au comportement de l'animal : ponte, aversion pour les chevaux, techniques de combat, etc. Barthélemy l'Anglais témoigne donc d'un intérêt pour des informations d'un autre type que Thomas de Cantimpré et affiche sa prédilection pour une source bien spécifique en fonction de chaque registre. Néanmoins, il ne s'en tient pas uniquement à des citations littérales, mais réalise des enchainements d'idées plus ou moins habiles, ce qui rend le texte moins transparent. Par exemple, il décrit la capacité de l'autruche à digérer le fer, puis insère librement une explication que nous n'avons pas retrouvée ailleurs: la dureté de la coquille de l'œuf permettrait de résister à cette forte chaleur naturelle. Plus loin, il justifie le fait que l'autruche dresse ses ailes pour faire fuir les chevaux en précisant qu'elle a pour eux une aversion, alors que l'Experimentator et Thomas de Cantimpré signale l'information de façon neutre sans indication de contexte (« alas erigit »)

\section{L'autruche dans le Speculum naturale de Vincent de Beauvais}

17 Vincent de Beauvais consacre quant à lui deux notices successives à l'autruche : de struthione et de eodem ${ }^{59}$. La première débute, comme chez Barthélemy l'Anglais, par la citation d'Isidore (1. 1-5), qui est cette fois beaucoup plus fidèle au texte source, sans remaniement ni ajout. Puis vient une citation du livre de Job (1. 5-7), qu'on retrouve par ailleurs dans les recueils de propriétés, suivie d'une citation du Physiologus (1. 7-18), elle aussi littérale mais écourtée. Ainsi, ces parties du texte source n'ont pas été conservées: «Hoc ergo animal, cum venerit tempus suum ut ova pariat, elevat oculos suos in caelum et videt si stella illa quae dicitur Virgilia ascendit » (« donc cet animal, à chaque fois que vient le moment de pondre ses œufs, lève les yeux vers le ciel et voit si s'élève cette étoile qui est appelée Virgilia ») et «De qua stella dicit Hiob: Qui facit Virgiliam et septentrionalem et dextrum et promptuaria austri [Hiob 9.9] ( Job dit de cette étoile : il fabrique Virgilia, le Septentrion, l'Orient et les constellations du Sud »). Ensuite, nous trouvons un passage Auctor (1. 18-21) qui englobe des données 
communément partagées dans les textes sources : le fait que l'autruche dresse ses ailes, qu'elle soit incapable de voler, qu'elle ait un corps immense et de rares plumes, des ailes semblables à celles du héron et de l'autour (Grégoire le Grand, Glose Ordinaire, Hugues de Fouilloy, Angelus, Angelus purus, Arbor abies alta, Anonyme de Clairvaux, Experimentator, Thomas de Cantimpré, Barthélemy l'Anglais), et qu'elle consomme du fer (Lambert de Saint-Omer, Alexander Neckam, Experimentator, Thomas de Cantimpré, Barthélemy l'Anglais).

La première des deux notices se termine par une longue citation d'Aristote (1.21-35), qui énonce les points communs et les différences entre l'autruche et le reste des oiseaux. La citation est fidèle à la source et reprend la grande majorité des critères : présence d'ailes qui ne permettent pas de voler et qui s'apparentent plus à des poils, paupières supérieures et cils, plumage important dans la partie inférieure, pattes au nombre de deux terminées par un ongle fendu, grande taille. Par contre, nous n'avons pas retrouvé ailleurs la fin de l'extrait, dont le contenu s'apparente à une réflexion d'ordre physiologique et médical : «Tres habet digitos in utroque pede propter alas, et propter pondus. Cumque sit ponderosi corporis et non boni volatus, multam habet superfluitatem, multumque coit, sicut et gallina et perdix » (1.32-35, « Elle a trois doigts à chaque pied en raison de ses ailes et en raison de son poids. Et puisque son corps est pesant et que son vol n'est pas bon, elle présente un grand surplus [superfluitas] et copule beaucoup, comme la poule et la perdrix »). La deuxième notice commence par un long extrait du Liber de natura rerum (1. 1-22), puis introduit une citation du Physiologus (1. 22-28), absente chez les autres compilateurs, qui souligne la qualité de la vue de l'autruche lui permettant de retrouver ses petits dans le sable. Enfin, il ajoute une citation du médecin arabe Rāzī (1. 28-29), qu'il est également le seul à utiliser.

Par rapport aux autres compilateurs, Vincent de Beauvais est très soucieux de respecter la littéralité du texte cité et d'utiliser le plus grand nombre de sources. Dans le Speculum naturale, les références aux auteurs sources jouent un rôle essentiel dans la structuration du propos, puisque Vincent de Beauvais préfère généralement, dans la version trifaria, ne pas scinder ni remanier le texte. Pas plus que Thomas de Cantimpré et Barthélemy l'Anglais, Vincent de Beauvais ne recourt à Pierre le Mangeur, Gervais de Tilbury et Hildegarde de Bingen. Parmi les auteurs arabes, on pourrait s'étonner de ne retrouver que la courte citation de Rāzī, et non les passages issus d'Isaac Israeli et d'Avicenne. Ce constat peut cependant s'expliquer aisément. Isaac Israeli ne consacre pas de notice spécifique à l'autruche : il était donc nécessaire de parcourir les chapitres sur les oiseaux pour y glaner çà et là quelques informations pertinentes. Dans le cas d'Avicenne, on n'a jusqu'à présent aucun indice permettant de supposer que Vincent de Beauvais ait utilisé l'Abbreviatio de animalibus, au contraire de Barthélemy l'Anglais. Quant au Canon, il faisait certes partie des sources régulièrement employées dans le Speculum naturale, mais le chapitre sur l'autruche n'y était pas classé sous la dénomination latine traditionnelle de l'animal, mais sous la transcription du terme arabe pour le désigner (de naham), ce qui explique que le passage ait résisté au repérage des frères dominicains.

\section{L'autruche chez Albert le Grand}

Afin de compléter ce panorama des synthèses naturalistes produites au sein des ordres mendiants, il convient enfin de se pencher sur les développements propres à la 
philosophie scolastique en s'attardant sur l'important commentaire réalisé par Albert le Grand sur l'œuvre d'Aristote. Dans son De animalibus, l'autruche est à la fois mentionnée dans le commentaire suivi sur l'ouvrage d'Aristote et dans la partie finale qui reprend un catalogue d'espèces animales sous forme de notices inspirées de Thomas de Cantimpré. Dans la partie de commentaire proprement dit, Albert le Grand résume à la suite d'Aristote les traits physiques qui permettent de situer l'autruche entre l'oiseau et le quadrupède : plumes dans le bas du corps et deux pattes comme les oiseaux, mais terminées par deux sabots fendus comme les quadrupèdes, taille assimilable à celle des quadrupèdes qui l'empêche de voler, rares plumes, duvet composé d'une sorte de poils très fins, présence de paupières supérieures ${ }^{60}$. Ailleurs, Albert reprend encore ce qui la distingue des autres oiseaux: à l'exception de l'autruche, tous les oiseaux ont quatre doigts ${ }^{61}$. Par rapport à Aristote, Albert apporte une précision nouvelle: comme la grue, la cigogne et d'autres oiseaux de ce type, l'autruche présente un long œsophage, dans lequel la nourriture séjourne longuement et descend petit à petit, afin d'être amollie et réchauffée ${ }^{62}$. Enfin, concernant le milieu géographique, Albert mentionne une espèce propre à la $\mathrm{Nubie}^{63} \mathrm{et}$, plus loin dans sa notice sur l'autruche, il reprend à Aristote l'idée que l'autruche évolue dans les déserts de Libye ${ }^{64}$.

Dans le catalogue final des différentes catégories d'animaux ${ }^{65}$, le chapitre sur l'autruche se fonde à la fois sur l'analyse d'Aristote et sur la reprise de contenus du Liber de natura rerum de Thomas de Cantimpré, complétées par un travail d'observation personnelle clairement revendiqué par Albert le Grand en fin de notice : «Voilà ce que j'ai vu de l'autruche, qui m'a semblé être non pas tant un oiseau [avis] qu'un intermédiaire entre le quadrupède [gressibile] et le volatile [volatile]. ${ }^{66}$ D'une part, Thomas de Cantimpré offre donc à Albert un relais vers des savoirs déjà présents chez Pline (stupidité de l'autruche, vitesse à la course) et dans le Physiologus (étymologie grecque renvoyant au fait que l'autruche a deux doigts à chaque pied comme le chameau). Par ailleurs, Albert intègre d'autres contenus introduits par Thomas de Cantimpré, puis repris par Vincent de Beauvais : peau épaisse qui protège l'autruche du froid et présence de petits os sous les ailes. D'autre part, les observations et expériences attestées par Albert - qu'il les ait réalisées de lui-même ou en soit du moins un témoin direct ou indirect - aboutissent à l'introduction de savoirs nouveaux, en particulier dans le cadre de la description anatomique de l'animal et dans l'optique de démentir de fausses croyances.

Parmi les auteurs étudiés, Albert est aussi le premier à détailler l'évolution du plumage en fonction de l'âge. En effet, il assure que la jeune autruche possède d'abord un plumage de couleur cendrée et que l'ensemble de son corps est alors recouvert de fines plumes. Mais à partir de la deuxième année, celui-ci se dénude progressivement en perdant les plumes des hanches, du cou et de la tête. La description morphologique est beaucoup plus précise et objective chez Albert, qui ajoute une estimation chiffrée de sa taille (cinq ou six pieds) et détaille davantage de traits physiques : tête d'oie, bec très petit par rapport à la dimension du corps, plumes du dos d'un noir intense, hanches très larges et cuisses charnues à la peau blanche. Enfin, Albert remet en doute la véracité de certains lieux communs sur l'autruche, qu'il dément sur la base d'expériences. Ainsi, il rapporte avoir proposé du fer à plusieurs autruches; aucune n'en a consommé, mais elles ont dévoré goulûment des os broyés et séchés, ainsi que des cailloux. Par ailleurs, il dément la rumeur selon laquelle l'autruche couverait ses œufs d'un simple regard, en expliquant que l'autruche pond ses œufs en juillet, les 
cache dans le sable avant leur éclosion grâce à la chaleur du soleil, et surveille régulièrement l'endroit où ils sont placés ${ }^{67}$.

\section{Interprétation des données}

Sur la base du tableau comparatif des sources fourni en annexe, plusieurs constats ressortent avec plus ou moins de force. Tout d'abord, certains traits physiques ne sont presque jamais décrits. Ainsi, la description de l'autruche ne reprend pas des éléments importants comme la forme du bec, avant Barthélemy l'Anglais, qui mentionne succinctement la présence du bec sans le décrire, puis surtout Albert le Grand, qui est le premier à donner une indication de proportion de sa taille par rapport au reste du corps. De même, une comparaison entre le mâle et la femelle n'est envisagée nulle part, alors que la couleur du plumage et la taille diffèrent entre les deux sexes. Nous ne trouvons pas non plus d'indication sur la durée de vie de l'animal, ni sur le temps d'éclosion des œufs. Le milieu géographique est peu présent dans les descriptions ; sa mention se limite à ce que l'on peut déduire de l'appellation « moineau de Libye » chez Aristote, aux indications de Pline et à l'évocation très vague de l'Afrique. À nouveau, c'est chez Albert le Grand qu'on trouve une information plus complète et retravaillée, mentionnant en outre la présence occasionnelle de l'animal dans la région de l'auteur.

Les traits descriptifs mentionnés par une seule source sont rares ; même l'anecdote sur l'autruche du roi Salomon, rapportée par Gervais de Tilbury, est en réalité héritée de Pierre le Mangeur. À l'inverse, aucune propriété ne traverse l'ensemble de la tradition. L'élément le plus souvent évoqué concerne l'habitude qu'a l'autruche d'enfouir ses œufs dans le sable. Ce constat reflète une volonté de décrire la natura de l'animal en accentuant les traits remarquables qui différencient l'autruche des autres espèces et qui sont solidement attestés dans la tradition. Cet élément s'inscrit dans la double perspective d'une description d'ordre physiologique et d'une exploitation moralisante ou symbolique. De même, le comportement de l'autruche vis-à-vis de son petit a été très remarqué, car également susceptible d'alimenter des interprétations typologiques. Par contre, les étapes de développement de l'autruchon sont passées sous silence dans l'ensemble des sources, à l'exception de l'évolution de la couleur du plumage remarquée par Albert le Grand sur la base d'une observation directe.

Les compilateurs se caractérisent également par leur volonté d'expliquer pourquoi l'autruche ne couve pas ses œufs. Sur ce point, parmi les sources potentielles, seule Hildegarde de Bingen avait évoqué avant eux la nature trop chaude de l'autruche, mais elle ne fait probablement pas partie des sources directement utilisées par les compilateurs. Le Liber de natura rerum reflète cependant une connaissance de certains passages qui sont aussi chez Hildegarde de Bingen, ce qui n'est pas le cas chez Barthélemy l'Anglais et Vincent de Beauvais. Thomas de Cantimpré pourrait bien avoir introduit cet élément, repris ensuite par les autres, alors que Barthélemy l'Anglais est le seul à mettre en rapport la grande taille et la dureté des œufs avec la nécessité de protéger le fotus d'une chaleur excessive pendant son développement. Ce souci d'expliquer les données observées sous l'angle physiologique s'inscrit dans une réflexion médicale de plus en plus manifeste au fil du XIIIe siècle, en lien avec les questions traitées dans les facultés de médecine qui commencent à se développer. Par contre, il est à noter qu'aucun auteur n'a remis en cause l'absence de couvaison (pas 
même Albert le Grand), alors que dans la réalité la femelle et le mâle s'alternent jour et nuit pour couver le nid, enfoui à une trentaine de centimètres sous le sable.

Si on concentre l'analyse sur les continuités propres aux encyclopédies des XIIe et XIIIe siècles, on aperçoit que la consommation de fer par l'autruche a été reprise systématiquement par chacun des auteurs envisagés: Lambert de Saint-Omer, Alexander Neckam, Thomas de Cantimpré, Barthélemy l'Anglais, Vincent de Beauvais, Albert le Grand. En dehors de cette particularité, il est remarquable que le régime alimentaire soit évoqué sous son aspect légendaire plutôt que sur un plan scientifique, à l'exception de l'expérience d'Albert le Grand qui remet cette croyance en question. Ce constat est à nouveau représentatif, dans les encyclopédies, d'un traitement de la natura de l'oiseau par sélection des traits remarquables, susceptibles aussi de faire l'objet d'une utilisation typologique. Cette perspective correspond à l'intention assumée par les compilateurs, d'offrir matière à d'éventuelles moralisations qui, sans être explicites, sont suggérées à partir des éléments décrits. Ainsi, il n'est pas directement fait mention de la correspondance avec l'hypocrisie, alors qu'il s'agit d'un élément majeur dans les sources et qui devait donc être bien connu des compilateurs. La comparaison entre les encyclopédistes permet aussi de relever cette exception : seul Vincent de Beauvais reprend des indications pharmacologiques, qui sont aussi absentes du discours scolastique d'Albert le Grand. Ce fait s'explique de plusieurs façons. Premièrement, dans le Canon d'Avicenne, l'autruche est mentionnée sous son nom arabe, la notice était donc très difficile à repérer. Deuxièmement, Vincent de Beauvais introduit de très nombreuses nouvelles sources médicales ${ }^{68}$, parmi lesquelles Rāzì. Cet apport reflète sans doute la disponibilité de cette auctoritas dans les bibliothèques fréquentées par les frères dominicains qui ont contribué au Speculum maius, mais il traduit aussi un intérêt plus grand pour une pharmacologie validée par les autorités médicales, et non plus seulement transmise dans les cercles monastiques. Par contraste, dans le livre sur l'anatomie humaine chez Thomas de Cantimpré, la plupart des recettes médicales sont fournies sans marqueur de citation précis.

Pour ce qui est des auteurs cités, la continuité est nette avec les autorités qui détiennent en quelque sorte un monopole dans leur registre spécifique. Ainsi, Isidore constitue l'autorité traditionnelle dans le champ de l'étymologie, tandis que le Physiologus domine au moment d'aborder les propriétés remarquables de l'autruche. Sur le plan des observations naturalistes, c'est plutôt Aristote - ainsi que ses commentateurs arabes - qui est privilégié pour entretenir la tendance à présenter l'autruche comme une espèce intermédiaire entre les oiseaux et les quadrupèdes. Cette particularité s'inscrit dans le cadre de l'échelle des êtres, bien que ce concept ne soit pas explicitement formulé par les descriptions. Ainsi, une attention spécifique est accordée dans la littérature médiévale aux espèces qui assurent la transition entre les différents échelons de cette hiérarchie naturelle. C'est par exemple le cas pour tracer la frontière entre l'humain et l'animal (quadrupède), avec le pygmée comme intermédiaire, dans l'exposé d'Albert le Grand et de Pierre d'Auvergne dans la seconde moitié du XIIIe siècle ${ }^{69}$. À l'autre bout de l'échelle de la nature, les coquillages marquent le passage entre le végétal et l'animal ${ }^{70}$. Les intermédiaires entre les autres catégories du vivant sont évoqués plus discrètement. Ainsi, de même que l'anguille assure la transition entre les reptiles et les poissons, ou encore la macreuse entre les poissons et les oiseaux ${ }^{71}$, l'autruche reflète quant à elle le passage progressif entre les oiseaux et les quadrupèdes. C'est sous cet angle qu'elle sera décrite anatomiquement, plutôt que dans 
une optique taxinomique éloignée de la perspective zoologique médiévale, mais qui prévaut de nos jours et montre les rapprochements physiques entre l'autruche et d'autres oiseaux qui lui sont apparentés.

L'analyse permet également de dégager d'importantes filiations entre les sources. Ainsi, Grégoire le Grand influence Isidore et, de là, toute la tradition spirituelle du XIIe siècle (dont la glose et les recueils de propriétés) et les encyclopédies du XIIIe siècle. Raban Maur complète Isidore, qui est sa source centrale, tandis qu'Avicenne prend logiquement son information chez Aristote sans introduire de nouvelles observations sur l'animal, si ce n'est d'ordre médical dans son Canon. Parmi les sources médicales d'origine arabe, Rāzì est le plus concis, mais sur base de ce premier élément (la nature grasse de la chair de l'autruche) se développent des considérations diététiques plus précises et étendues chez Isaac Israeli, puis chez Avicenne. Les recueils de propriétés à usage des prédicateurs et les encyclopédies des ordres mendiants entretiennent quant à eux d'étroites relations, qui se justifient du fait de s'adresser à un même lectorat. Parmi les dominicains, Thomas de Cantimpré a considérablement influencé Vincent de Beauvais et Albert le Grand, auxquels il sert d'intermédiaire vers d'autres sources plus anciennes, comme Pline et le Physiologus. Ainsi, c'est bien souvent la disponibilité d'une information et non le choix du compilateur qui explique la présence de tel ou tel contenu.

En revanche, il convient de mettre en évidence les moments où un compilateur choisit de s'écarter d'une source qu'il connaît pourtant et utilise ailleurs dans son texte. On remarque ainsi que Barthélemy l'Anglais ne recourt quasiment pas à Pline l'Ancien ${ }^{72}$, donnant la préférence à Aristote, dont il reprend une majeure partie des éléments, mais pas la description de la tête de l'autruche (tête pelée, paupières et cils, long cou). En comparaison, Thomas de Cantimpré suit assez peu Aristote dans ce chapitre et fait un usage plus important de Pline, mais avant tout pour les traits de comportement (régime alimentaire, enfouissement du cou dans le sable, lancement de pierres contre les assaillants), et non pour évoquer le milieu géographique ni pour décrire l'anatomie de l'animal (sauf pour mentionner la présence de paupières). Il est alors fréquent que Vincent de Beauvais reprenne ces passages d'Aristote qui avaient été écartés par Thomas de Cantimpré : description des pattes, duvet comme des poils, ailes servant à la course.

Dans ce panorama, certains auteurs se distinguent enfin par leur originalité : c'est le cas d'Hildegarde de Bingen, dont les sources restent difficilement identifiables, mais aussi, dans une certaine mesure, de Thomas de Cantimpré, qui introduit des éléments nouveaux comme l'aversion de l'autruche envers le cheval, de Barthélemy l'Anglais qui ajoute l'explication selon laquelle la taille et la dureté des œufs permettent de protéger le fœetus pendant son développement, et d'Albert le Grand, qui rapporte plusieurs observations et expériences personnelles afin de compléter et de vérifier le propos qu'il reprend majoritairement d'Aristote et de Thomas de Cantimpré.

\section{Conclusion}

$\mathrm{Au}$ terme de ce parcours au cœur des sources et des textes compilés, revenons rapidement sur la façon dont Claudia Radogna situait les compilations médiévales dans le développement des savoirs relatifs aux animaux. Selon elle, " pour une histoire de la zoologie, il serait intéressant de considérer les livres des encyclopédies consacrées aux 
animaux comme le domaine d'une science qui, au fur et à mesure qu'elle se développe, se détache du symbolique, du monstrueux, du fabuleux et du fantastique pour s'approcher lentement d'une vue sur le monde animal qui tente au moins d'en comprendre les lois internes. Et tout cela, non seulement grâce aux textes de la tradition arabe d'Aristote et de Pline, mais aussi en vertu d'une indépendance renouvelée de la ratio, qui se manifeste déjà à la fin du XIIe siècle comme mouvement interne à la culture occidentale et chrétienne ${ }^{73}$. L'idée de considérer les encyclopédies comme un reflet de l'évolution de l'état des savoirs zoologiques est en effet pertinente. Cependant, comme on a pu le constater avec le cas de l'autruche, l'image qu'elles renvoient n'est pas tant celle d'une évolution caractérisée par une rationalisation croissante, mais plutôt celle d'une science cumulative, fondée sur une juxtaposition habile de savoirs issus de registres variés. En effet, les chapitres sur l'autruche montrent que ces différentes approches de sa nature et ses propriétés se côtoient et s'additionnent l'une à l'autre, sans que l'une ne remplace l'autre, pour offrir une description synthétique sur l'animal, considéré à la fois dans ses dimensions objective et symbolique. Ainsi, dans le monde latin du XIIIe siècle, la science sur les animaux cherche à associer ces deux versants avec cohérence, ce qui nécessite un important travail de sélection des sources pour construire un exposé encyclopédique. En comparaison, la synthèse produite par Albert le Grand dans un cadre scolastique poursuit d'autres finalités ; reposant avant tout sur Aristote, elle intègre néanmoins de nombreux éléments repris de Thomas de Cantimpré, qui sont parfois remis en cause au gré de nouvelles observations et expériences.

En ce sens, l'écriture naturaliste médiévale répond à plusieurs enjeux épistémologiques, d'une part vis-à-vis des sources citées plus ou moins fidèlement, d'autre part vis-à-vis des savoirs véhiculés, écartés ou - plus rarement - nouvellement introduits. Thomas de Cantimpré s'octroie ainsi davantage de liberté par rapport à ses sources, qu'il cite parfois de façon imprécise, ce qui lui permet en contre-partie d'introduire des originalités en intervenant librement sur le texte. En comparaison, Barthélemy l'Anglais reformule légèrement le texte source, mais plutôt dans la perspective de renforcer l'articulation interne de son propos. Chez lui, le recours successif aux différentes autorités répond au besoin d'aborder des sujets bien déterminés en fonction des sources qu'il choisit de privilégier, comme Isidore qui est préféré au Physiologus pour l'étymologie et Aristote à Pline pour les descriptions anatomiques. Quant à Vincent de Beauvais, il est beaucoup plus sensible à préserver la littéralité des passages cités, qu'il reprend sous forme de blocs successifs. Les interventions personnelles sont très limitées, le but étant d'offrir un exposé qui repose sur un plus grand nombre de sources, y compris dans le domaine pharmacologique. Chez lui, la sélection et l'alternance des autorités citées contribuent fortement à la structuration. Enfin, Albert le Grand, qui poursuit un but d'explication philosophique et rationnelle du vivant, s'inscrit dans une double continuité par rapport à Aristote et à Thomas de Cantimpré, par l'intermédiaire duquel il intègre des contenus présents chez Pline ou dans le Physiologus. L'observation personnelle joue un rôle beaucoup plus important et permet de préciser la description anatomique de l'animal et de démentir certaines rumeurs erronées.

Sur le plan des savoirs diffusés, si les apports personnels des compilateurs sont peu nombreux, la volonté d'apporter des explications raisonnées aux phénomènes observés est notable, par exemple pour justifier l'enfouissement des œufs de l'autruche dans le 
sable. Thomas de Cantimpré, qui fut un prédicateur itinérant très actif, accorde une grande importance aux traits susceptibles d'exploitation homilétique. Barthélemy l'Anglais présente quant à lui le souci de construire un chapitre très structuré autour de thèmes de prédilection, comme l'étymologie ${ }^{74}$, la classification et la description physique de l'animal, et son comportement. Enfin, les sources médicales entrent de plus en plus en considération, en particulier chez Vincent de Beauvais, qui introduit abondamment les sources arabes d'Avicenne et de Rāzī, au moment où elles commencent à s'introduire dans les programmes des universités occidentales ${ }^{75}$.

En somme, la méthodologie que nous avons adoptée, consistant à partir des sources potentielles pour étudier ensuite leur intégration dans les chapitres compilés, a permis de se placer dans la position du compilateur latin devant un nombre important de sources dans des registres très diversifiés ${ }^{76}$. Dans cette optique, l'auteur source n'est alors plus envisagé en fonction des seules citations dont il fait l'objet, mais également de ce qui n'en a pas été conservé dans les encyclopédies. Il concentre dès lors la pleine attention d'une recherche qui porte directement sur sa réception au sein des encyclopédies latines et, par corollaire, sur son assimilation par un lectorat élargi, en parallèle du développement de la culture universitaire au milieu du XIIIe siècle.

\section{I. Éditions de textes}

ALBERTUS MAGNUS, De animalibus libri XXVI : nach der Cölner Urschrift, STADLER Hermann, (éd.), Münster, Aschendorff, 1916 (Beiträge zur Geschichte der Philosophie des Mittelalters, 15-16). ARISTOTELES, De animalibus. Michael Scot's Arabic-Latin Translation, vol. II, Books XI-XIV: Parts of Animals., VAN OPPERAAIJ Aafke M.I., Leiden - Boston - Köln, 1998 (Aristoteles Semitico-Latinus 5.2). ARISTOTELES, De animalibus. Michael Scot's Arabic-Latin Translation, III, Books XV-XIX: Generation of Animals, VAN OPPENRAAIJ Aafke M.I., avec un index grec au De Generatione animalium par DROSSAART LULOFS H.J., Leiden - New York - Köln, 1992 (Aristoteles Semitico-Latinus 5.3).

AVICENNA, De animalibus per magistrum Michaelem Scotum de Arabico in Latinum translatus, DE' GREGORI Giovanni e Gregorio (éd.), Venise, 1500.

AVICENNA, Liber Canonis, Venise, 1507, reprod. G. Olms, Hildesheim, 1964.

BARTHOLOMAEUS ANGLICUS, De proprietatibus rerum= Bartholomaei Anglici de genuinis rerum caelestium, terrestrium et inferarum proprietatibus libri XVIII, Frankfurt, 1601.

Biblia cum glossa ordinaria, pars II, Rusch Adolf (éd.), Strasbourg, 1481.

BRUNETTO LATINI, Li Livres dou Tresor, BALDWIN Spurgeon W. et BARRETTE Paul (éds.), Tempe, Arizona, 2003. 
Dicta Chrysostomi= WILHELM FRIEDRICH, « Der ältere und der jungere Physiologus », in ID., Denkmäler deutscher Prosa des 11. Und 12. Jahrhunderts, 2 vol. , München, 1914-1916, t. II, p. 17-44.

Experimentator= DEUS Janine, Der "Experimentator" - eine anonyme lateinische Naturenzyklopädie des frühen 13. Jahrhunders, thèse doctorale, Université de Hambourg, 1998.

GERVASIUS TILBERIENSIS, Otia imperialia= Gervase of Tilbury, Otia imperialia: Recreation for an Emperor, éd. BANKs Shelagh E. et BINNS James W. (éd.), Oxford, Clarendon Press, 2002 (Oxford Medieval Texts).

GESNER Conrad, Historiae Animalium Liber III, qui est de Avium natura, WECHELUS Ioannes (éd.), Frankfurt, 1585.

GREGORIUS MAGNUS, Moralia in Iob = Sancti Gregorii Magni Moralium Libri, pars quarta, Migne Jean-Paul (éd.), (Patrologia Latina, 76), col. 9-780.

HILDEGARDIS BINGENSIS, Physica= Hildegardis Bingensis Physica, MIGNE Jean-Paul (éd.), (Patrologia Latina, 197), col. 1117-1352.

HUGUES DE FOUILLOY, Aviarium= The Medieval Book of Birds. Hugh of Fouilloy's Aviarium, CLARK Willene B. (éd.), Binghamton - New York, 1992.

ISAAC ISRAELI, De dietis = Omnia opera Ysaac, Lyon, 1515, pars prima, f. 11r - f. 156r.

ISIDORE DE SÉVILLE, Etymologies XII. Des animaux, ANDRÉ Jacques (éd.), Paris, Les Belles Lettres, 1984.

LAMBERTUS AUDOMARENSIS, Liber floridus = Lambertus Audomarensis Liber Floridus, éd. DEROLEZ Albert, Gent, 1968.

NECKAM Alexander, De naturis rerum = Alexandri Neckam De naturis rerum libri duo with the poem of the same author De Laudibus divinae sapientiae, WRIGHT Thomas (éd.), London, Longman, Roberts, and Green, 1863 (Rerum Britannicarum Medii Aevi Scriptores, or Chronicles and Memorials of Great Britain and Ireland during the Middle ages, 34).

PETRUS COMESTOR, Historia scholastica, MIGNE Jean-Paul (éd.), (Patrologia latina, 198), col. 1053-1722.

Physiologus latinus. Versio B, CARMODY Francis J., Paris, 1939.

PIERRE DE BEAUVAIS, Le Bestiaire. Version longue attribuée à Pierre de Beauvais, BAKER Craig (éd.), Paris, 2010.

PLINE L'ANCIEN, Histoire naturelle, X, DE SAINT-DENIS Eugène (éd.), Paris, Les Belles Lettres, 1961.

RABAN MAUR, De universo = Beati Rabani Mauri De universo libri XXII, Migne Jean-Paul (éd.), (Patrologia latina, 111), col. 9-614.

RHAZES, Liber Rasis ad Almansorem, in Contenta in hoc volumine. Liber Rasis ad Almansorem, Divisiones eiusdem, Liber de iuncturarum egritudinibus eiusdem, Liber de egritudinibus puerorum eiusdem [...], LoCATELLus Bonetus (éd.), Venise, 1497.

THOMAS CANTIMPRATENSIS, Liber de natura rerum, BOESE Helmut (éd.), Berlin - New York, de Gruyter, 1973.

VINCENTIUS BELLOVACENSIS, Speculum naturale, Douai, 1624. Version en ligne : http:// sourcencyme.irht.cnrs.fr/encyclopedie/voir/133 


\section{Travaux}

BÉNATOUïL Thomas, « Mouvements et vie chez Aristote : quelques remarques « autour » des plantes », Anais de Filosofia Clássica, 13/25, 2019, p. 1-20 〈hal-02300126〉.

CHANDELIER Joël, Avicenne et la médecine en Italie. Le Canon dans les universités (1200-1350), Paris, Champion, 2017.

CIPRIANI Mattia, La place de Thomas de Cantimpré dans l'encyclopédisme médiéval : les sources du Liber de natura rerum, Thèse de doctorat, Paris-Florence, EPHE - Scuola Normale superiore (Istituto Italiano di Scienze Umane), 2 tomes, 2014.

CIPRIANI Mattia, « 'In dorso colorem habet inter viridem et ceruleum'... Liber rerum e osservazione zoologica diretta nell'enciclopedia di Tommaso di Cantimpré », Reinardus, 29, 2018, p. 16-98.

CLESSE Grégory, « Un compilateur en eaux (in-)connues. Thomas de Cantimpré et la faune aquatique du Nord-Ouest de l'Europe », Anthropozoologica, 53 (7), 2018, p. 87-96. DOI : 10.5252/ anthropozoologica2018v53a7.

URL : http://anthropozoologica.com/53/7.

CLESSE Grégory et NINITTE Florence, « Semblables aux fils d'Adam : les êtres entre humanité et animalité, de la cosmographie à l'écriture scientifique et encyclopédique de langue arabe », in SCIANCALEPORE Antonella (dir.), Corps hybrides aux frontières de l'humain au Moyen Âge, Institut d'Études Médiévales de l'Université Catholique de Louvain, Louvain-la-Neuve, 2020, p. 131-151.

CLESSE Grégory, « De la source au confluent : l'usage des ouvrages médicaux d'origine arabe par les compilateurs latins du XIIIe siècle », in VAN DEN ABEELE Baudouin, DE CALLATAŸ Godefroid, CAVAGNA Mattia et VAN HAEPEREN Françoise (dir.), Bilan et perspectives des études sur les encyclopédies médiévales, Institut d'Études Médiévales de l'Université Catholique de Louvain, Louvain-la-Neuve, Université catholique de Louvain (Publications de l'Institut d'études médiévales, à paraître).

DRAELANTS Isabelle et FRUNZEANU Eduard, « Le savoir astronomique et ses sources dans le De mundo et corporibus celestibus de Barthélemy l'Anglais », Rursus-Spicae [en ligne], 11, 2017.

DOI: $10.4000 /$ rursus. 1352

URL: http://journals.openedition.org/rursus/1352

Косн Joseph, « Sind die Pygmäen Menschen ? Ein Kapitel aus der philosophischen Anthropologie der mittelalterlichen Scholastik », Archiv für Geschichte der Philosophie, 40, 1931, p. 194-213.

KÖHLER Theodor Wolfram, « Anthropologische Erkennungsmerkmale menschlichen Seins. Die Frage der "Pygmei" in der Hochscholastik », in ZIMMERMANN Albert et SPEER Andreas (dir.), Mensch und Natur im Mittelalter, vol. 2, New York - Berlin, 1992, p. 718-735.

KUHRY Emmanuelle, « Dictionnaires, distinctions, recueils de propriétés en milieu cistercien. Outils pour la prédication, sources pour l'étude de la nature ", in FALMAGNE Thomas, STUTZMANN DOminique, TURCAN-VERKERK Anne-Marie (dir.), Les cisterciens et la transmission des textes (XIIe-XVIIIe siècles), Turnhout, Brepols, 2018, p. 285-338.

KUHRY Emmanuelle, «Panorama des manuscrits et nouvelles ressources pour l'étude de la tradition manuscrite du Physiologus latin », RursuSpicae [en ligne], 2, 2019.

DOI :10.4000/rursuspicae.924

URL : http://journals.openedition.org/rursuspicae/924

LONATI Elisa, « Plinio il Vecchio e Vincenzo di Beauvais : Quale modello di Naturalis Historia per lo Speculum Maius? », Filologia Mediolatina, 25, 2018, p. 323-352. 
LONATI Elisa, « Bartolomeo Anglico, "the English Pliny” ? ", Archivum Latinitatis Medii Aevi, 76, 2018, p. 223-278.

Louis Pierre, « Animaux anonymes chez Aristote », Bulletin de l'Association Guillaume Budé, 2, 1971, p. 211-217.

MAILLARD Ninon, « Être « de la nature du poisson » sans être « véritable » poisson : le cas de la macreuse, oiseau comme animal, poisson comme chère ", Revue semestrielle de droit animalier, Université de Limoges, 2017, p. 263-280.

MCCULLOCH Florence, Mediaeval Latin and French Bestiaries, University of North Carolina Press, Chapel Hill, 1960.

PAULMIER-FOUCART Monique, avec la collaboration de DUCHENNE Marie-Christine, Vincent de Beauvais et le Grand miroir du monde, Brepols, Turnhout, 2004.

RADOGNA Claudia, « La zoologie médiévale : le crocodile entre historia et ratio », in CASSIN Barbara, LABARRIÈRE Jean-Louis et ROMEYER DHERBEY Gilbert (dir.), L'animal dans l'Antiquité, Paris, Vrin, 1997, p. 519-531.

RoLING Bernd, Physica Sacra: Wunder, Naturwissenschaft und historischer Schriftsinn zwischen Mittelalter und Früher Neuzeit, Leiden - Boston, Brill, 2013.

SCHULER Stefan, « Medicina secunda philosophia. Die Einordnung der Medizin als Hauptdisziplin und die Zusammenstellung ihrer Quellen im Speculum maius des Vinzenz von Beauvais », Frühmittelalterliche Studien, 33, 1999, p. 169-251.

VAN DEN ABEELE Baudouin, «Vincent de Beauvais naturaliste : les sources des livres d'animaux du Speculum naturale », in LUSIGNAN Serge, PAULMIER-FOUCART Monique et DUCHENNE Marie-Christine (dir.), Lector et compilator. Vincent de Beauvais, frère prêcheur. Un intellectuel et son milieu au XIIIe siècle, Grâne, 1997, p. 127-151.

VAN DEN ABEELE Baudouin, «L'allégorie animale dans les encyclopédies latines du Moyen Age », in BERLIOZ Jacques, POLO DE BEAULIEU Marie-Anne et COLlOMB Pascal (dir.), L'animal exemplaire au Moyen Age (Ve-XVe siècles), Rennes, 1999, p. 123-143.

VAN DEN ABEELE Baudouin, « Mauvais merles et faucons exemplaires : ambivalences dans la symbolique des oiseaux au Moyen Age », in MAZOYER Michel, PÉREZ REY Jorge, MALBRAN-LABAT Florence et LEBRUN René (dir.), L'oiseau, entre ciel et terre, Paris, 2005, p. 331-351.

WILLE Clara, « Der Reiher, das Neunauge und der Igel. Tiernamen im romanischen Mittelalter », in OBERMAIER Sabine (dir.), Tiere und Fabelwesen im Mittelalter, Berlin - New York, 2009, p. 79-101.

\section{NOTES}

1. Nous tenons à remercier Isabelle Draelants, Emmanuelle Kuhry, Baudouin Van den Abeele et Mattia Cipriani pour leurs apports d'information, leurs suggestions et les précieux conseils dont cet article a bénéficié.

2. GESNER C., Historiae Animalium Liber III, qui est de Avium natura, WECHELUS Ioannes (éd.), Frankfurt, 1585 : p. 740 (représentation de l'autruche) et p. 743, 1. 29-32 (citation) : Ova mense Iulio parit, et in sabulo abscondit, quae calore solis excuduntur, ut alia multa ova animalium. Et ideo ad eam non revertitur, quod nudo corpore ea ea fovere non possit; et (subinde) respicit ad locum in quo condita sunt, unde falso aliqui crediderunt quod visu ea foveat, Albert. de anim. lib. 23. 
3. CLESSE G., «Un compilateur en eaux (in-)connues. Thomas de Cantimpré et la faune aquatique du Nord-Ouest de l'Europe ", Anthropozoologica, 53 (7), 2018, p. 87-96. Voir aussi ID., " De la source au confluent: l'usage des ouvrages médicaux d'origine arabe par les compilateurs latins du XIIIe siècle ", in VAN DEN ABEelE B., DE CALlataÿ G., CAVAGNA M. et Van HAEPEREN F. (dir.), Bilan et perspectives des études sur les encyclopédies médiévales, Institut d'Études Médiévales de l'Université Catholique de Louvain, Louvain-la-Neuve (à paraître).

4. Sur les sources utilisées dans les livres sur les animaux, voir entre autres la mise au point récente sur l'usage de sources non traditionnelles par Thomas de Cantimpré : CIPRIANI M., "'In dorso colorem habet inter viridem et ceruleum'... Liber rerum e osservazione zoologica diretta nell'enciclopedia di Tommaso di Cantimpré ", Reinardus, 29, 2018, p. 16-98. Pour les nombreuses études sur l'encyclopédisme médiéval, nous renvoyons à la bibliographie proposée sur le site de l'Atelier Vincent de Beauvais: https://ateliervdb.hypotheses.org/bibliographie-surlencyclopedisme-medieval.

5. Nous suivons ici la typologie des sources esquissée par VAN DEN ABEELE B., «Vincent de Beauvais naturaliste : les sources des livres d'animaux du Speculum naturale », in Lector et compilator. Vincent de Beauvais, frère prêcheur. Un intellectuel et son milieu au XIIIe siècle, LUSIGNAN S., PAULMIER-FOUCART M. et DUCHENNE M.-C. (dir.), Grâne, 1997, p. 127-151. Nous remercions par ailleurs Baudouin Van den Abeele d'avoir aimablement mis à notre disposition les extraits relatifs à l'autruche qu'il avait déjà recueillis dans les traditions des bestiaires et des encyclopédies.

6. Les éditions utilisées sont les suivantes, par ordre chronologique des œuvres: PLINE L'ANCIEN, Histoire Naturelle, Livre X, DE SAINT-DENIS E. (éd.), Les Belles Lettres, Paris, 1961 (autruche : livre X, sections 1-2, 56, 143, p. 28, 47, 77) ; ISIDORE DE SÉvILLE, Étymologies XII. Des animauX, ANDRÉ J. (éd.), Les Belles Lettres, Paris, 1984 (autruche : livre XII, section 7, 20, p. 238); RABAN MAUR, De universo libri XXII, éd. Patrologia latina, t.111, col. 9-614 (autruche: livre VIII, c. 6, col. 245); LAMBERTUS AUDOMARENSIS, Liber Floridus, DEROLEZ A. (éd.), Gent, 1968 (autruche : c. 46) ; HILDEGARDIS BINGENSIS, Physica, éd. P.L., t. 197, Paris, 1855, col. 1117-1352 ; ALEXANDER NECKAM, De naturis rerum, WRIGHT T. (éd.), London, 1863 (autruche : distincio I, c. 50, p. 101).

7. Pour une synthèse récente sur le contenu et les fluctuations propres à la tradition des bestiaires, voir KUHRY E., «Panorama des manuscrits et nouvelles ressources pour l'étude de la tradition manuscrite du Physiologus latin », RursuSpicae [en ligne], 2, 2019.

8. Les éditions utilisées sont les suivantes: Physiologus latinus. Versio B, CARMODY F.J. (éd.), Paris, 1939 (autruche : c. 27, p. 48) ; pour les dicta Chrysostomi, wiLhelm F., « Der ältere und der jungere Physiologus", in ID., Denkmäler deutscher Prosa des 11. Und 12. Jahrhunderts, 2 vol., München, 1914-1916, t. II, p. 17-44 (autruche: c. 24, p. 40); Hugues de Fouilloy, Aviarium= The Medieval Book of Birds. Hugh of Fouilloy's Aviarium, CLARK W.B. (éd.), Binghamton - New York, 1992 (autruche: c. 42, p. 188-198).

9. Sur les recueils de propriétés, voir KUHRY E., « Dictionnaires, distinctions, recueils de propriétés en milieu cistercien. Outils pour la prédication, sources pour l'étude de la nature », in FALMAGNE T., STUTZMANN D., TURCAN-VERKERK A.-M. (dir.), Les cisterciens et la transmission des textes (XIIe-XVIIIe siècles), Turnhout, Brepols, 2018, p. 285-338.

10. Ces textes sont consultés dans les éditions et manuscrits suivants : GREGORIUS MAGNUS, Moralium Libri, pars quarta, (éd. P.L., t. 76), livre XXXI, c. 8-12, col. 578-582 ; Biblia cum glossa ordinaria, pars II, RUSCH A. (éd.), Strasbourg, 1481, f. 224r ; GARNIER DE ROCHEFORT (attrib.), Distinctiones Angelus, ms. Troyes, BM 392, f. 144r; Angelus purus natura : ms. Montpellier, Bibl. de l'école de médecine, H 470, f. $100 \mathrm{v}$ et Paris, B.n.F. lat. 14297, f. 179v; Abies arbor alta: ms. Bruxelles, KBR II 1060, f. 120r ; Anonyme de Clairvaux: ms. Troyes, B.M. 1236, f. 89v.

11. Le texte du Liber ad Almansorem est consulté dans l'édition suivante: RHAZES, Liber Rasis ad Almansorem, in Contenta in hoc volumine. Liber Rasis ad Almansorem, Divisiones eiusdem, Liber de iuncturarum egritudinibus eiusdem, Liber de egritudinibus puerorum eiusdem [...], LoCATELLUS B. (éd.), 
Venise, 1497 (autruche : tract. III, c. 10, 1. 15-16, f. 12va). Nous consultons le De dietis d'Isaac Israeli dans l'édition suivante : ISAAC ISRAELI, De dietis, in Omnia opera Ysaac, Lyon, 1515, pars prima, f. 11r f. $156 \mathrm{r}$ (autruche : Diete univ., c. 48-53, f. 75v-f. 78r). Pour l'Abbreviatio de animalibus d'Avicenne, nous utilisons l'édition suivante: AVICENNA, De animalibus per magistrum Michaelem Scotum de Arabico in Latinum translatus, DE' GREGORI G. (éd.), Venise, 1500 (autruche : livre XIV, c. De anathomia mamillarum et labiorum, f. 47r). Enfin, pour son Canon, nous nous fondons sur l'édition suivante : AVICENNA, Liber Canonis, Venise, 1507, reprod. G. Olms, Hildesheim, 1964 (autruche : Liber Canonis, II, 526, f. 138v).

12. Physiologus latinus, Versio B, c. 27 : p. 48.

13. WILHELM, « Der ältere und der jungere Physiologus », 1914-1916, t. II, c. 40: 40.

14. ISIDORE, Etym., XII,7, $20: 236$.

15. RABAN MAUR, De universo, VIII, $6:$ col. 245.

16. AVICENNA, Liber Canonis, II, 526 : f. $138 \mathrm{v}$.

17. ARISTOTELES, De animalibus. Michael Scot's Arabic-Latin Translation, II, Books XI-XIV : Parts of Animals., VAN OPPERAAIJ A.M.I. (éd.), Leiden - Boston - Köln, 1998 (autruche : PA, III, 14,697b 14-26).

18. AVICENNA, De animalibus : f. $47 \mathrm{r}$.

19. PLINE L'ANCIEN, Histoire Naturelle, $X, 1: 28$.

20. HUGUES DE FOUILLOY, Aviarium, $42: 188-198$.

21. Physiologus latinus. Versio B, $27: 48$.

22. En latin: herodius et accipiter, parfois aussi traduit par «épervier ». Sur le sens de herodius, terme désignant initialement le héron, qui en vint ensuite à désigner le faucon, voire le seul gerfaut, voir wiLlE C., "Der Reiher, das Neunauge und der Igel. Tiernamen im romanischen Mittelalter ", in Tiere und Fabelwesen im Mittelalter, OBERMAIER S. (éd.), Berlin - New York, 2009, p. 79-101.

23. GREGORIUS MAGNUS, Moralia, XXXI, 8-12 : col. 578 ; Biblia cum glossa ordinaria : f. 224r ; LAMBERTUS AUDOMARENSIS, Liber Floridus, 46 ; HUGUES DE FOUILLOY, Aviarium, c. 42 : 188-198; GARNIER DE ROCHEFORT (attrib.), Distinctiones Angelus, ms. Troyes, B.M. 392, f. 144r ; Angelus purus natura : ms. Montpellier, Bibl. de l'école de médecine, H 470, f. 100v et Paris, B.n.F lat. 14297, f. 179v; Abies arbor alta: ms. Bruxelles, KBR II 1060, f. 120r ; Anonyme de Clairvaux : ms. Troyes, B.M. 1236, f. 89v.

24. Physiologus latinus. Versio B, c. $27: 48$.

25. HILDEGARDIS BINGENSIS, Physica : col. 1117-1352.

26. ALEXANDER NECKAM, De naturis rerum : 101.

27. PLINE L'ANCIEN, Histoire Naturelle, X, 1:28; Lambertus Audomarensis, Liber Floridus, c. 46. Sur l'appellation de l'autruche comme "moineau de Libye» chez Aristote, voir LouIs Pierre, «Animaux anonymes chez Aristote », Bulletin de l'Association Guillaume Budé, 2, 1971, p. 211-217.

28. PLINE L'ANCIEN, Histoire Naturelle, X, $1: 28$; HILDEGARDIS BINGENSIS, Physica : col. 1117-1352.

29. GERVASIUS TILBERIENSIS, Otia imperialia, III, 104, in Gervase of Tilbury, Otia imperialia. Recreation for an Emperor, BANKS S.E. \& BINNS J.W. (éd.), Oxford, 2002, p. 790-791.

30. PETRUS COMESTOR, Historia scholastica, III Reg., c. 8 (éd. P.L., t. 198), Paris, 1855, col. 1353-1354.

31. PLINE L'ANCIEN, Histoire Naturelle, X, 1: 28; LAMBERTUS AUDOMARENSIS, Liber Floridus, c. 46 ; ALEXANDER NECKAM, De naturis rerum : 101.

32. HILDEGARDIS BINGENSIS, Physica : col. 1117-1352.

33. ARISTOTELES, De animalibus. Michael Scot's Arabic-Latin Translation, III, Books XV-XIX : Generation of Animals, VAN OPPENRAAIJ A.M.I., avec un index grec au De Generatione animalium par DROSSAART LULOFS H.J., Leiden - New York - Köln, 1992 (autruche : GA, III, 1, 749b17).

34. ISIDORE DE SÉvILLE, Etym., XII, 7, 20 : 238; RABAN MAUR, De universo: col. 245 ; LAMBERTUS Audomarensis, Liber Floridus, c. 46 ; Physiologus latinus. Versio B, $27: 48$; wilhelm, «Der ältere und der jungere Physiologus », 1914-1916, c. 24 : 40 ; GREGoRIUS MAGNUS, Moralia, XXXI, 8-12 : col. 578 ; 
Biblia cum glossa ordinaria, pars II : f. 224r ; HUGUES DE FOUILLOY, Aviarium, c. 42 : 188-198; GARNIER DE ROCHEFORT (attrib.), Distinctiones Angelus, ms. Troyes, B.M. 392, f. 144r ; Angelus purus natura: ms. Montpellier, Bibl. de l'école de médecine, H 470, f. 100v et Paris, B.n.F. lat. 14297, f. 179v ; Abies arbor alta : ms. Bruxelles, KBR II 1060, f. 120r ; Anonyme de Clairvaux : ms. Troyes, B.M. 1236, f. 89v ; HILDEGARDIS BINGENSIS, Physica : col. 1117-1352.

35. GREGORIUS MAGNUS, Moralia, XXXI, 8-12: col. 578; Biblia cum glossa ordinaria, pars II: f. 224r; HUGUES DE FOUILLOY, Aviarium, c. 42: 188-198.

36. MCCUlLOCH F., Mediaeval Latin and French Bestiaries, University of North Carolina Press, Chapel Hill, 1960, p. 146.

37. VAN DEN ABEELE B., « Mauvais merles et faucons exemplaires : ambivalences dans la symbolique des oiseaux au Moyen Age », in Mazoyer M., PÉREZ REY J., MALBRAN-LABAT F. et LEBRUN R. (dir.), L'oiseau, entre ciel et terre, L'Harmattan, Paris, 2005, p. 332-342. En effet, le merle dispose d'un chant mélodieux qui ravit l'homme, mais sa noirceur peut évoquer le péché Le paon, quant à lui, est associé à la vanité, mais est aussi image du Christ en raison de la qualité de conservation de sa chair qui est une image de l'immortalité. Enfin, le faucon est dans certains cas symbole de sobriété, parfois associé aux saints et même au Christ, dans la mesure où il revient au fauconnier attiré par un morceau de chair, tout comme les pécheurs sont attirés par l'image des plaies du Christ. Mais il est aussi symbole des vanités terrestres et se fait l'attribut de certains vices, comme la haine, l'envie, l'orgueil.

38. VAN DEN ABEELE B., "L'allégorie animale dans les encyclopédies latines du Moyen Age ", in BERLIOZ J., POlO DE BEAUlieu M.-A. et COLlomb P. (dir.), L'animal exemplaire au Moyen Age (Ve - XVe siècles), Rennes, 1999, p. 123-143.

39. Physiologus latinus. Versio B, c. $27: 48$.

40. PLINE L'ANCIEN, Histoire Naturelle, $\mathrm{X}, 1: 28$.

41. GREGoRIUS MAGNUS, Moralia, XXXI, 8-12 : col. 578 ; Biblia cum glossa ordinaria, pars II : f. 224r ; HUGUES DE FOUILLOY, Aviarium, c. 42 : 188-198 ; Anonyme de Clairvaux : ms. Troyes, B.M. 1236, f. 89v.

42. RABAN MAUR, De universo : col. 245.

43. ALEXANDER NECKAM, De naturis rerum : 101.

44. PLINE L'ANCIEN, Histoire Naturelle, $\mathrm{X}, 1: 28$.

45. HILDEGARDIS BINGENSIS, Physica: col. 1117-1352.

46. RHAZES, Liber ad Almansorem, III, 10 : f. 12va.

47. ISAAC ISRAELI, Diete univ., c. 48-53 : f. 75v-78r.

48. AVICENNA, Liber Canonis, II, $526:$ f. 138v.

49. THOMAS CANTIMPRANENSIS, Liber de natura rerum, V, 110, BOESE H. (éd.), p. 226-227.

50. CIPRIANI M., La place de Thomas de Cantimpré dans l'encyclopédisme médiéval : les sources du Liber de natura rerum, Thèse de doctorat, Paris-Florence, EPHE - Scuola Normale superiore (Istituto Italiano di Scienze Umane), 2 tomes, 2014 (voir vol. I, 211 et vol. II, p. 207-209).

51. La propriété est rapportée dans les encyclopédies de Thomas de Cantimpré, Barthélemy l'Anglais, Vincent de Beauvais, Albert le Grand et Arnold de Saxe. Elle se trouve aussi dans la version longue du bestiaire attribuée jusqu'il y a peu à Pierre de Beauvais (Le Bestiaire. Version longue attribuée à Pierre de Beauvais, BAKER C. (éd.), Paris, 2010, c. 29, p. 117), ainsi que dans le Livre $d u$ trésor de Brunet Latin: BRUNETTO LATINI, Li Livres dou Tresor, BALDWIN S. et BARRETTE P. (éd.),Tempe, Arizona, 2003, p. 133). À partir des XIIe-XIIIe siècles, le fer à cheval dans le bec constitue d'ailleurs l'attribut iconographique traditionnel de l'autruche, comme nous le signale Baudouin Van den Abeele; cet attribut se maintiendra aux Temps Modernes, par son passage dans les Emblemata de la Renaissance. À propos de la reprise de propriétés médiévales d'animaux chez les auteurs renaissants, voir entre autres RoLING B., Physica Sacra: Wunder, Naturwissenschaft und historischer Schriftsinn zwischen Mittelalter und Früher Neuzeit, Leiden - Boston, Brill, 2013. 
52. Experimentator, 4.55, dans DEUS J., Der "Experimentator" - eine anonyme lateinische Naturenzyklopädie des frühen 13. Jahrhunders, thèse doctorale, Université de Hambourg, 1998, p. 319 : In eundo alas erigit et quibusdam aculeis acutis naturaliter sub alis insertis se ipsam pungit et sic ire compellit. Calidissime est nature ita, quod ferrum deglutit et digerit. Equum naturaliter odit et infestat et in tantum ei contrariatur, ut equs [sic] eam uidere non possit.

53. VAN DEN ABEELE, "L'allégorie animale dans les encyclopédies latines du Moyen Âge », 1999 : 123-143.

54. Pour une étude approfondie sur la diffusion du Canon d'Avicenne dans le Moyen Âge occidental, voir CHANDELIER J., Avicenne et la médecine en Italie. Le Canon dans les universités (1200-1350), Paris, Champion, 2017, spéc. 35-78 sur la répartition des manuscrits conservant la traduction latine du Canon.

55. BARTHOLOMAEUSANGLICUS, Liber de proprietatibus rerum, Frankfurt, 1601, XII, $33: 547$.

56. Experimentator, $4.55: 319$.

57. Experimentator, $4.55: 319$; THOMAS CANTIMPRANENSIS, LDNR, $5.110: 226-227$.

58. Experimentator, $4.55: 319$; THOMAS CANTIMPRANENSIS, LDNR, $5.110: 226-227$.

59. VINCENTIUS BELLOVACENSIS, Speculum naturale, Douai, 1624, XVI, 138-139 : col. 1231-1232.

60. AlBERTUS MAGNUS, De animalibus libri XXVI : nach der Cölner Urschrift, STADLER H. (éd.), Münster, Aschendorff, $1916: 989$.

61. ALBERTUS MAGNUS, De animalibus : 983.

62. ALBERTUS MAGNUS, De animalibus: 929.

63. ALBERTUS MAGNUS, De animalibus : 983.

64. ALBERTUS MAGNUS, De animalibus : 1510 .

65. ALBERTUS MAGNUS, De animalibus : 1510.

66. ALBERTUS MAGNUS, De animalibus : 1510 : Haec sunt quae vidi de strutione quae non tam avis quam medium inter gressibile et volatile esse michi videtur.

67. ALBERTUS MAGNUS, De animalibus : 1510.

68. Sur l'inflation de la médecine dans le Speculum naturale et dans le Speculum doctrinale, voir SCHULER S., «Medicina secunda philosophia. Die Einordnung der Medizin als Hauptdisziplin und die Zusammenstellung ihrer Quellen im Speculum maius des Vinzenz von Beauvais", Frühmittelalterliche Studien, 33, 1999, p. 169-251. Sur les sources et l'évolution des contenus chez Vincent de Beauvais, voir aussi PAULMIER-FOUCART M., avec la collaboration de DUCHENNE M.-C, Vincent de Beauvais et le Grand miroir du monde, Brepols, Turnhout, 2004 : 44-104.

69. CLESSE G. et NINITTE F., « Semblables aux fils d'Adam : les êtres entre humanité et animalité, de la cosmographie à l'écriture scientifique et encyclopédique de langue arabe ", in SCIANCALEPORE A. (dir.), Corps hybrides aux frontières de l'humain au Moyen Âge, Institut d'Études Médiévales de l'Université Catholique de Louvain, Louvain-la-Neuve, 2020, p. 131-151. Voir aussi косн J., "Sind die Pygmäen Menschen? Ein Kapitel aus der philosophischen Anthropologie der mittelalterlichen Scholastik », Archiv für Geschichte der Philosophie, 40 1931, p. 194-213; KÖHLER T.W., «Anthropologische Erkennungsmerkmale menschlichen Seins. Die Frage der "Pygmei" in der Hochscholastik », in ZIMMERMANN A. et SPEER A. (dir.), Mensch und Natur im Mittelalter, vol. 2, , New York - Berlin, de Gruyter, 1992, p. 718-735.

70. BÉNATOUïL T., "Mouvements et vie chez Aristote: quelques remarques «autour» des plantes », Anais de Filosofia Clássica, 13/25, 2019, p. 1-20 〈hal-02300126〉.

71. MAILLARD N., "Être « de la nature du poisson » sans être "véritable» poisson: le cas de la macreuse, oiseau comme animal, poisson comme chère", Revue semestrielle de droit animalier, Université de Limoges, 2017, p. 263-280.

72. Sur l'usage de la Naturalis Historia de Pline par Barthélemy l'Anglais et par Vincent de Beauvais, nous renvoyons à LONATI E., "Bartolomeo Anglico, "the English Pliny"?», Archivum 
Latinitatis Medii Aevi, 76, 2018, p. 223-278 ; EAD., «Plinio il Vecchio e Vincenzo di Beauvais : Quale modello di Naturalis Historia per lo Speculum Maius ? ", Filologia Mediolatina, 25, 2018, p. 323-352.

73. RADOGNA C., «La zoologie médiévale: le crocodile entre historia et ratio", in CASSIN B., LABARRIÈRE J.-L. et ROMEYER DHERBEY Gilbert (dir.), L'animal dans l'Antiquité, Paris, Vrin, 1997, p. 526-527.

74. Il s'agit là aussi d'un trait typique de l'Experimentator, qui s'inspire d'Isidore de Séville. De peu antérieur au De proprietatibus rerum, ce texte constitue une source utilisée régulièrement par Barthélemy l'Anglais, sans être mentionnée, cf. DRAELANTS I. et FRUNZEANU E., «Le savoir astronomique et ses sources dans le De mundo et corporibus celestibus de Barthélemy l'Anglais ", Rursus-Spicae [en ligne], 11, 2017, spéc. 6-12.

75. Sur la diffusion manuscrite de la traduction latine du Canon d'Avicenne : CHANDELIER, Avicenne et la médecine en Italie. Le Canon dans les universités (1200-1350), 2017 : 35-78.

76. Cette entreprise implique néanmoins une connaissance approfondie du réseau des bibliothèques visitées par chacun des compilateurs et de leur contenu. En complément, une enquête approfondie sur l'histoire des bibliothèques serait souhaitable pour confirmer et préciser les résultats obtenus.

\section{RÉSUMÉS}

Le plus souvent, l'étude des sources encyclopédiques tend à partir des compilations pour remonter le courant vers les autorités qui les ont alimentées. Cet article, sur les contenus relatifs à l'autruche, propose la démarche inverse. Dans un premier temps, il s'agit d'établir une typologie des savoirs zoologiques disponibles sur cet animal au XIIIe siècle, en parcourant un vaste éventail de sources dans des registres variés : ouvrages d'histoire naturelle de l'Antiquité avec Aristote et Pline, littérature de moralisation, synthèses encyclopédiques, recueils de propriétés, traités médicaux. Sur cette base se fonde dans un second temps l'analyse de la réception et de l'assimilation effective de ces informations dans les principales synthèses naturalistes des ordres mendiants au XIIIe siècle, en se focalisant sur le Liber de natura rerum de Thomas de Cantimpré, le De proprietatibus rerum de Barthélemy l'Anglais, le Speculum naturale de Vincent de Beauvais et le De animalibus d'Albert le Grand. L'article interroge les critères qui soustendent la sélection opérée par ces auteurs, en prêtant une attention spécifique non seulement aux savoirs qui ont été relayés, mais aussi à ceux qui ont été écartés ou nouvellement introduits.

Most of the time, the study of encyclopaedic sources tends to start with the compilations to lead to the authorities which are quoted. This article, focusing on the ostrich, proposes the opposite approach. As a first step, we establish a typology of the zoological knowledge available in the 13th century on this animal. We do this, considering a wide range of sources in various disciplines: works of natural history in the Antiquity with Aristotle and Pliny, moral literature, encyclopedic syntheses, alphabetical series of properties, and medical treatises. The second step is to analyse the reception and assimilation of these contents in the main compendia of natural science produced by the mendicant orders in the middle of the thirteenth century, focusing on the Liber de natura rerum of Thomas of Cantimpré, the De proprietatibus rerum of Bartholomaeus Anglicus, the Speculum naturale of Vincent of Beauvais, and the De animalibus of Albert the Great. With this article we wish to contribute to the question of the criteria underlying the selection 
made by these authors, paying specific attention to the transmitted knowledge as well as to what is rejected or introduced in an original way.

INDEX

Mots-clés : encyclopédies médiévales, zoologie, autruche, étude des sources, XIIIe siècle Keywords : Medieval Encyclopaedias, Zoology, Ostrich, Study of Sources, Thirteenth Century

\section{AUTEUR}

\section{GRÉGORY CLESSE}

Grégory Clesse (UCLouvain, Institut INCAL) est actuellement chargé de recherches au F.N.R.S. Après avoir réalisé une thèse de doctorat portant sur l'usage des sources arabes dans les compilations latines du XIIIe siècle, il concentre à présent l'essentiel de ses recherches sur la transmission d'Aristote et des auteurs arabes dans la zoologie et la médecine médiévale. 